\title{
Assessing the Effect of Laboratory Activities on Core Curricular Units of an Engineering Master's Program: A Multivariate Analysis
}

\author{
António M. Lopes $\mathbb{D D}^{1,2}$ Lucas da Silva $\mathbb{D D}^{1,2}$ and Jorge Seabra $\mathbb{D}^{1,2}$ \\ ${ }^{1}$ Departamento de Engenharia Mecânica, Faculdade de Engenharia, Universidade do Porto, Porto, Portugal \\ ${ }^{2}$ Institute of Science and Innovation in Mechanical and Industrial Engineering (INEGI), Porto, Portugal \\ Correspondence should be addressed to António M. Lopes; aml@fe.up.pt
}

Received 7 December 2020; Accepted 21 May 2021; Published 2 June 2021

Academic Editor: Adrian Neagu

Copyright (c) 2021 António M. Lopes et al. This is an open access article distributed under the Creative Commons Attribution License, which permits unrestricted use, distribution, and reproduction in any medium, provided the original work is properly cited.

\begin{abstract}
This paper studies the effect of laboratory activities on the results obtained by the students on 7 core curricular units (CUs) of an Integrated Master in Mechanical Engineering. Each CU is characterized by means of 4 performance indices, over the 6-year period 2014/2015-2019/2020. Firstly, individual indices in a per semester basis are compared. Secondly, the CUs are regarded as objects defined in a 4-dimensional space of features, and the multidimensional scaling (MDS) technique is adopted for clustering and computer visualization. The MDS is powerful for analyzing the multivariate dataset, unveiling patterns not perceived by standard statistical methods.
\end{abstract}

\section{Introduction}

Laboratory training is fundamental in engineering education [1-10], but understanding how the experimental activities contribute to meaningful learning and to achieve the goals established for a specific curricular unit (CU) is challenging [11-14]. Researchers often classify the educational engineering laboratories as follows: (i) hands-on with real equipment; (ii) simulated or virtual; and (iii) remote [15-19]. Despite this strict classification, many laboratory environments adopt a blended approach, where hands-on, virtual, and remote experiments are combined together, trying to simultaneously potentiate the advantages and mitigate the drawbacks of each one $[20,21]$. The laboratory experiments envisage strengthening: (i) the conceptual understanding, namely, the students' capabilities to understand and to solve problems related to the fundamental concepts presented in the theoretical classes; (ii) the design skills, meaning the students' skills to solve open-ended problems through the design and creation of new processes; (iii) the social skills, which mean the students' abilities to learn how to undertake engineering-related work in groups; and (iv) the professional skills, namely, the students' capabilities to become familiar with the technical expertise that they will need in their professional activities $[1,2]$. The best procedure to accomplish these four objectives in the context of undergraduate engineering programs is an open issue, which is hardened by multiple specific factors, as the teaching/ learning subjects, the students' backgrounds, or the number of students enrolled in the CU.

The Integrated Master in Mechanical Engineering (MIEM) at the Faculty of Engineering of the University of Porto (FEUP) prepares students so that, after completing the cycle of studies (CS), they can initiate a professional career in applied and/or research activities in Mechanical Engineering or related fields. To accomplish such objective, MIEM provides the students a solid educational framework with a rich scientific background and emphasis on the interaction between theory and practice within the main domains of Mechanical Engineering.

The importance of laboratory classes has always been recognized by those responsible for the CS, and these classes were implemented on most CUs of MIEM. In fact, the laboratory classes were early considered as an asset that allowed the students to acquire an important training component in technological areas and motivated them 
greatly to engage in the learning processes. The laboratory classes were also intended to give the students the opportunity to work in teams and an avenue to communicate their ideas in a concise, objective, and pragmatic way, as well as to develop their critical analysis of results. The increase in the laboratory component of the MIEM CUs was successively recommended by the accreditation bodies, namely, for the CUs in the areas of Applied Mechanics and Fluids and Energy, which are core areas of Mechanical Engineering. All CUs of these scientific areas included in the common part of the CS syllabus had no laboratory activities. In some of them, the students had extra difficulties, which translated in getting worse results than those verified in other CUs of the CS program. These CUs had in common the adoption of teaching methods based on theoretical classes for the exposition of subjects and theoretical-practical classes for problem solving. Moreover, they had a high number of enrolled students and high failure rates. To mitigate these issues, while avoiding changing the MIEM syllabus, which would have involved a slow and bureaucratic administrative process, the direction of the CS, with the support of the Mechanical Engineering Department (DEMec), decided to implement mandatory laboratory activities in some CUs of the second up to sixth semesters of MIEM, starting in the academic year 2016/2017. The procedure adopted, still in practice, requires that the students should carry out a set of laboratory works throughout the semester. Such activities are performed in small team groups, following the guidelines provided by the $\mathrm{CU}$ staff, with the objective of applying the knowledge acquired at the theoretical and theoreticalpractical classes. The laboratorial experiments should follow a script with precise instructions and be completed in a limited time, while the students are supported by monitors, who typically are students of the last semester of MIEM. The students are assessed via small written reports, one per experiment.

In this work the effect of laboratory activities on the results obtained by the students on 7 core CUs of MIEM is assessed. These CUs are Mechanics I, Solids Mechanics, Mechanics III $\}$, to be denoted by $\{$ MI, SM, MIII $\}$, and \{Thermodynamics II, Fluid Mechanics I, Fluid Mechanics II, Heat Transfer\}, to be denoted by \{TII, FMI, FMII, HT\}. The two sets belong to the areas of Applied Mechanics and Fluids and Energy, respectively. Each CU is characterized by the 4 performance indices \{passed/enrolled students' ratio, passed/assessed students' ratio, arithmetic mean of the passed students' marks, arithmetic mean of the assessed students' marks $\}$, to be denoted by $\{\mathrm{Pas} / \mathrm{Enr}$, Pas/Ass, Mean Pas, Mean Ass\}, over the 6-year period 2014/2015-2019/ 2020. Firstly, the performance indices characterizing each $\mathrm{CU}$ are compared with those of the CUs in the same semester, and their time evolution is analyzed in an individual basis. Secondly, the CUs in a given semester are considered objects defined in a 4-dimensional space of features, and the multidimensional scaling (MDS) technique is adopted for dimensionality reduction, clustering, and computer visualization of patterns. This is accomplished by choosing a suitable distance metric for object-to-object comparison and Procrustes analysis for obtaining meaningful maps of objects (that is, CUs) which are able to unveil possible hidden relationships between the data.

Having these ideas in mind, the paper organization is as follows. In Section 2, the MIEM program and the CUs are introduced briefly. In Section 3, a statistical study is performed in a per semester basis, to understand the time evolution of the results obtained in each CU. In Section 4, a multivariate analysis is conducted to unveil possible patterns embedded in the data, which are difficult or impossible to be noticed when adopting simple statistics. Finally, in Section 5, the main conclusions are summarized.

\section{The Curricular Units in the Context of the MIEM Program}

The current MIEM is a CS combining a first and a second cycle degrees into a single 5-year CS, corresponding to the bachelor ( 3 years) and to the master ( 2 years), respectively. The MIEM is organized in 10 semesters. At the end of the first cycle, the students have a solid base education, comprising the essential scientific and engineering skills and fundamental, nonspecialized knowledge in the various areas of Mechanical Engineering. After concluding the second cycle, the MIEM graduates have advanced level of training in Mechanical Engineering, focusing in Automation, Design and Manufacturing, Industrial Management, Mechanical Project and Construction, or Thermal Energy.

The 7 CUs of the MIEM program considered herein $\{\mathrm{MI}$, TII, SM, FMI, FMII, MIII, HT\} occur between the second and sixth semesters. Each semester includes 5 CUs in total, as follows:

(i) Second semester: \{Mathematical Analysis II, Computer Programming I, Statistics, Thermodynamics I, Mechanics I $\}$, to be referred as $S_{2}=\{$ MAII, CPI, S, TI, MI\}

(ii) Third semester: \{Mathematical Analysis III, Electricity, Numerical Analysis, Mechanics II, Thermodynamics II $\}$, to be referred as $S_{3}=\{$ MAIII, E, NA, MII, TII\}

(iii) Fourth semester: \{Metallic Materials, Mechanical Engineering Drawing, Instrumentation for Measurement, Solid Mechanics, Fluid Mechanics I\}, to be referred as $S_{4}=\{M M, M E D, I M, S M, F M I\}$

(iv) Fifth semester: \{Structural Mechanics I, Fluid Power Systems, Computer Aided Design and Manufacturing, Non Metallic Materials, Fluid Mechanics II $\}$, to be referred as $S_{5}=\{$ SMI, FPS, CADM, NMM, FMII\}

(v) Sixth semester: \{Manufacturing Processes I, Industrial Automation and Safety, Information Systems, Mechanics III, Heat Transfer $\}$, to be referred as $S_{6}=\{$ MPI, IAS, IS, MIII, HT $\}$

Table 1 summarizes a few details of the CUs, namely, the students/staff total contact hours and the total amount of time allocated to the $\mathrm{CU}$, regarding autonomous study, classes, and other academic activities. For thorough 
TABLE 1: The students/staff total contact hours and the total amount of time allocated to the 7 CUs.

\begin{tabular}{lccccc}
\hline \multirow{2}{*}{ CU } & Semester & Contact hours & \multicolumn{3}{c}{ Amount of time allocated (hours) } \\
& & & Autonomous study & Classes & Other activities \\
\hline MI & $\mathbf{S}_{2}$ & 58.5 & 106 & 56 & 0 \\
TII & $\mathbf{S}_{3}$ & 58.5 & 80 & 63 & 19 \\
SM & $\mathbf{S}_{4}$ & 58.5 & 95 & 63 & 4 \\
FMI & $\mathbf{S}_{4}$ & 58.5 & 93 & 59 & 6 \\
FMII & $\mathbf{S}_{5}$ & 58.5 & 101 & 63 & 6 \\
MIII & $\mathbf{S}_{6}$ & 58.5 & 108 & 54 & 0 \\
HT & $\mathbf{S}_{6}$ & & & 97 & 6 \\
\hline
\end{tabular}

information, please consult the FEUP's website at http:// www.fe.up.pt.

$M=7$ CUs in the set $\{$ MI, TII, SM, FMI, FMII, MIII, HT $\}$ and they have adopted identical teaching methods based on theoretical classes for subject exposition and theoreticalpractical classes for problem solving. Experimental activities have been negligible. The considerable numbers of students enrolled, between 202 and 275 in year 2019-2020, and the high failure rates in the CUs became a concern to the CS and DEMec directors. Therefore, in an attempt to relieve the problem, laboratory activities were adopted in 2016/2017, for \{MI, SM, MIII, FMII, HT\}, and in 2017/2018, for \{TII, FMI\}. Such laboratory activities require that the students should carry out predefined laboratory experiments throughout the semester. The work is performed in small groups, according to provided guidelines and in-loco staff support, which envisages consolidating the subjects taught in both the theoretical and the theoretical-practical classes.

\section{Statistical Analysis of the Students' Performance}

In this Section, the 4 performance indices $\{\mathrm{Pas} / \mathrm{Enr}$, Pas/Ass, Mean Pas, Mean Ass $\}$ that characterize each CU over the 6 academic years 2014/2015-2019/2020 are compared, in an individual basis, with those that characterize the CUs in the same semester. This means that MI is compared with the CUs in $\mathbf{S}_{2}$, TII with the CUs in $\mathbf{S}_{3}$, and so on, and the comparison involves one index at a time.

Figure 1 depicts the performance indices of the CUs versus time. The red lines represent the values of the performance indices of each indicated $\mathrm{CU}$, and the black lines represent the arithmetic means of the performance indices of the CUs in the same semester, either $\mathbf{S}_{2}, \ldots, \mathbf{S}_{6}$, respectively. The bottom and top edges of the blue boxes represent the 25 and 75 percentiles, while the central marks stand for the medians. The whiskers extend between the extreme data points, when excluding the outliers, and the outliers are represented by means of the symbol "o." We verify that most CUs' performance indices follow the trend exhibited by the CUs in their semester, over the entire period 2014/ 2015-2019/2020. This would mean that the effect of the laboratory activities is either negligible or hidden. However, some CUs reveal complex relationships for certain performance indices, as those depicted in the graphs denoted by (xii), (xiv), and (xv), and further analysis is needed.
To get more solid conclusions, we calculate the Pearson correlation $\rho_{\mathbf{x y}}$ as follows:

$$
\rho_{\mathrm{xy}}=\frac{\sum_{t=1}^{6}\left(x_{t}-\mu(\mathbf{x})\right) \cdot\left(y_{t}-\mu(\mathbf{y})\right)}{\sqrt{\sum_{t=1}^{6}\left(x_{t}-\mu(\mathbf{x})\right)^{2}} \cdot \sqrt{\sum_{t=1}^{6}\left(y_{t}-\mu(\mathbf{y})\right)^{2}}},
$$

where $\mathbf{x}=\left[x_{1}, \ldots, x_{6}\right]$ represents the values of a performance index $\{\mathrm{Pas} /$ Enr, Pas/Ass, Mean Pas, Mean Ass $\}$ for a given $\mathrm{CU}$ and $\mathbf{y}=\left[y_{1}, \ldots, y_{6}\right]$ stands for the arithmetic mean of the index for the CUs that belong to the same semester, in the 6-year period 2014/2015-2019/2020. The symbol $\mu(\bullet)$ denotes the arithmetic mean of the argument. Table 2 summarizes the results obtained. We verify that $\rho_{\mathbf{x y}}$ is high for most CUs, with the exception of $\{\mathrm{SM}, \mathrm{FMI}, \mathrm{MIII}\}$, which exhibit small values of $\rho_{\mathrm{xy}}$ for certain performance indices. This confirms the rough assumptions made previously through simple observation of the graphs since a low $\rho_{\mathbf{x y}}$ translates into discrepancies between the trends exhibited by a specific CU and the CUs in the same semester.

\section{Multivariate Analysis of the Students' Performance}

The analysis performed in Section 3 using simple statistics showed that not all performance indices lead to identical results. Therefore, examining the indices separately may reveal inconsistencies and bring the analysis inconclusive. To mitigate this issue, in the follow-up, a multivariate analysis is performed, meaning that all features characterizing the data are considered simultaneously.

Several mathematical techniques are available for multivariate data analysis. The principal component analysis (PCA) [22], additive trees [23], cluster analysis [24], factor analysis [25], and multiple regression analysis [26], among others [27], can be cited. Herein, the MDS [28-31] is adopted. MDS has advantages over other methods, such as PCA, which has difficulties to identify clusters surrounded by rings of points. Moreover, MDS is a more general projection method than PCA [28].

4.1. MDS Data Clustering and Visualization. MDS is a computational technique for visualizing information [28-31]. MDS detects meaningful fundamental dimensions that allow the researcher to visualize (dis)similarities between the objects of interest. Given $M$ objects in a $q$-dimensional space, the algorithm receives as input a $M \times M$ 


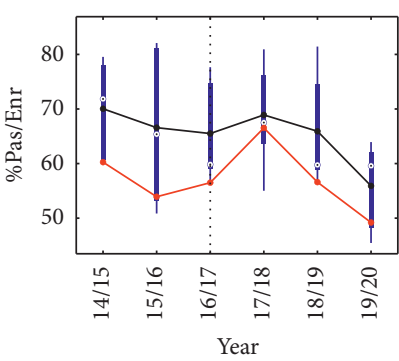

$\rightarrow$ Mean CUs in $S_{2}$

$\rightarrow$ MI

(a)

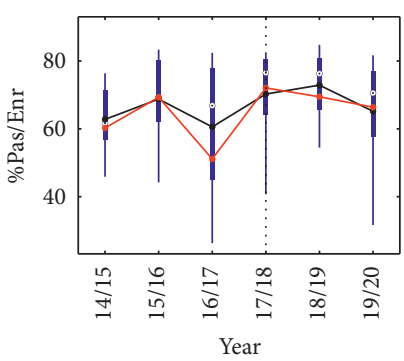

$\rightarrow$ Mean CUs in $S_{3}$

$\rightarrow$ TII

(e)

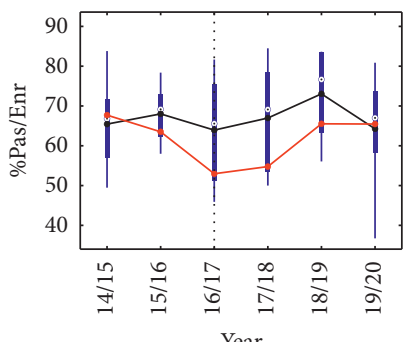

$\multimap$ Mean CUs in $S_{4}$

$\rightarrow$ SM

(i)

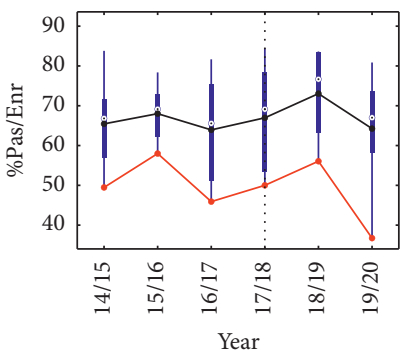

- Mean CUs in $S_{4}$

$\longrightarrow-$ FMI

(m)

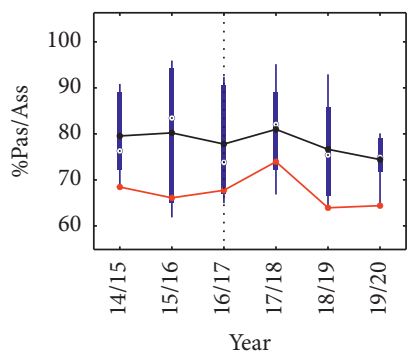

$\longrightarrow$ Mean CUs in $S_{2}$

(b)

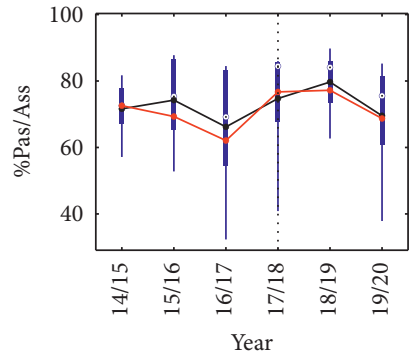

$\rightarrow$ Mean CUs in $S_{3}$

$\rightarrow$ TII

(f)

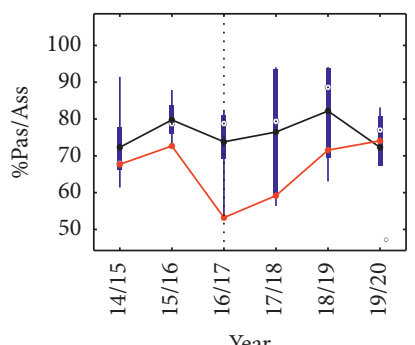

$\longrightarrow$ Mean CUs in $S_{4}$

(j)

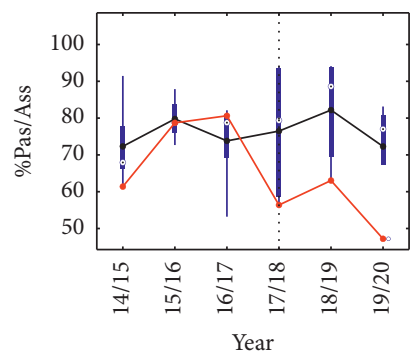

$\rightarrow$ Mean CUs in $S_{4}$

(n)

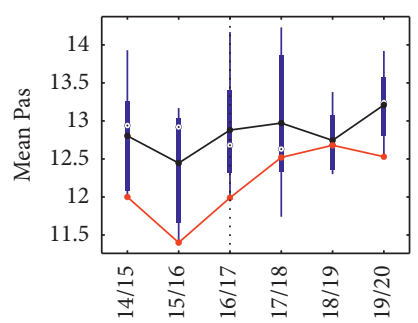

Year

$\longrightarrow$ Mean CUs in $S_{2}$
$\rightarrow$ MI

(c)

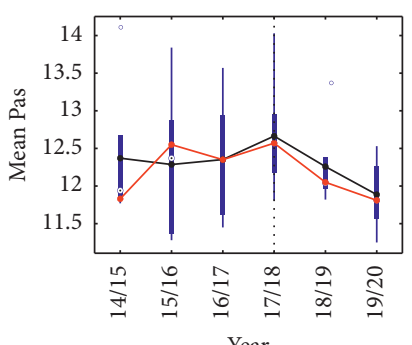

- Mean CUs in $S_{3}$

$\rightarrow$ TII

(g)

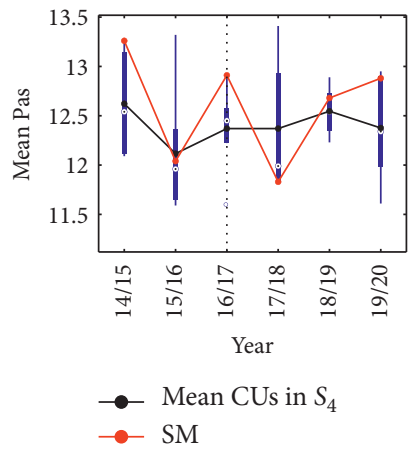

(k)

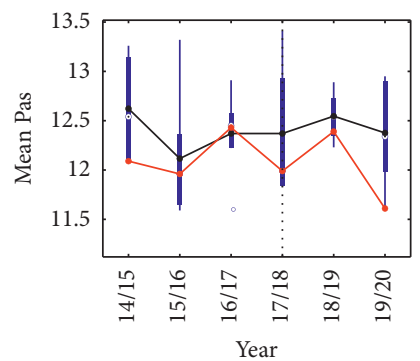

Mean CUs in $S_{4}$

(o)

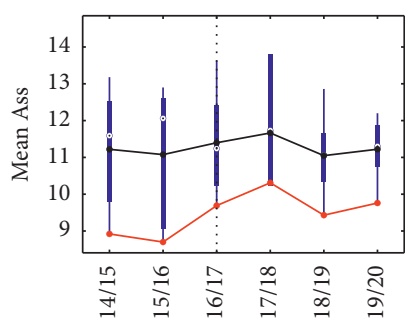

Year

$\rightarrow$ Mean CUs in $S_{2}$

(d)

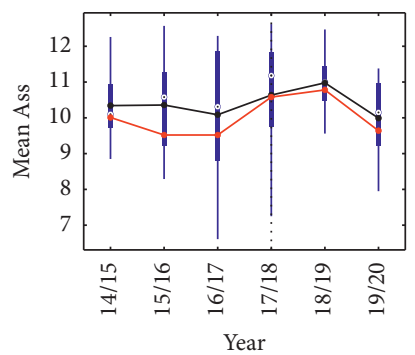

$\rightarrow$ Mean CUs in $S_{3}$

$\longrightarrow$ TII

(h)

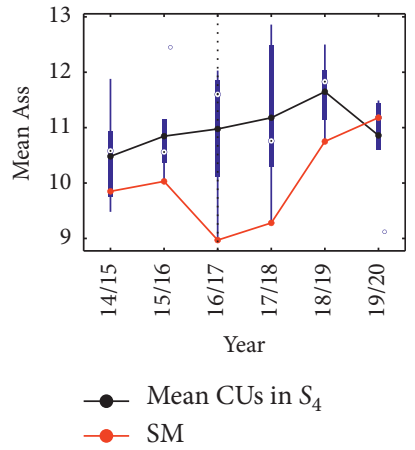

(1)

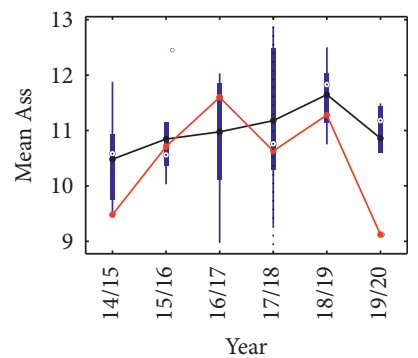

Mean CUs in $S_{4}$

$\longrightarrow$ FMI

(p)

Figure 1: Continued. 


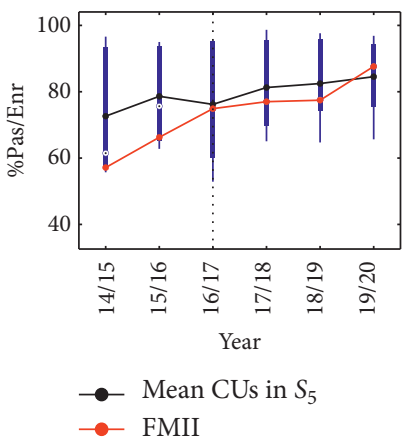

(q)

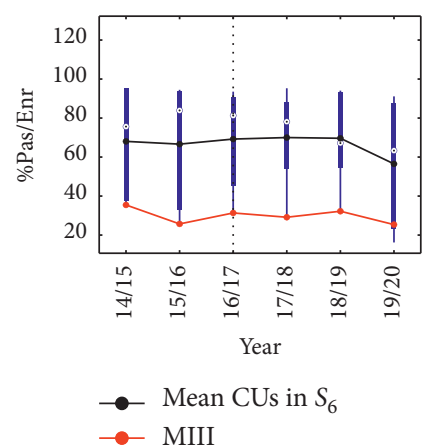

(u)

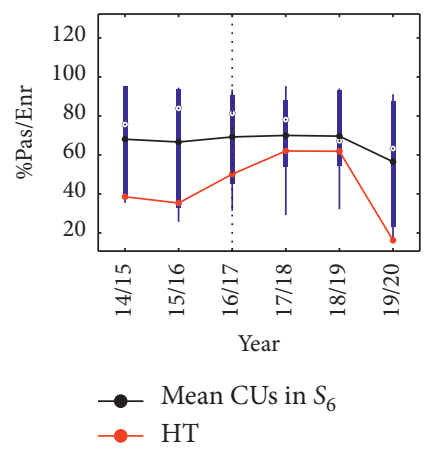

(y)

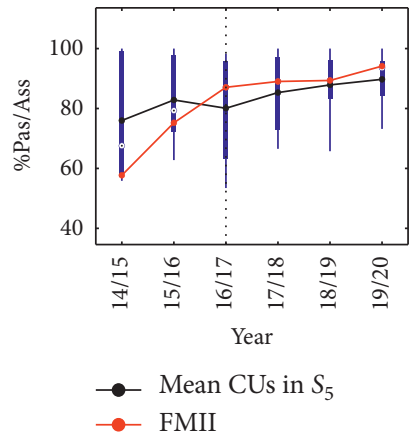

(r)

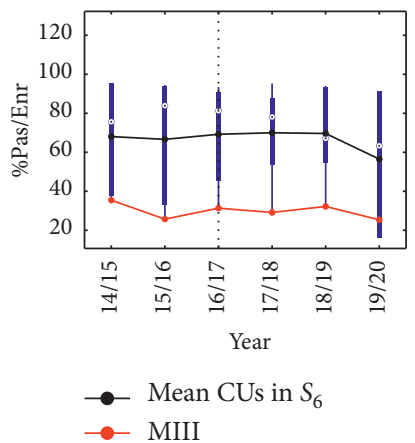

(v)

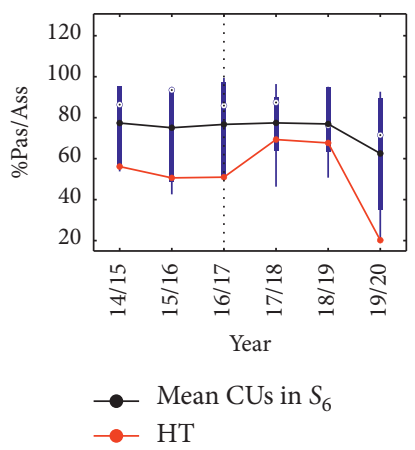

(z)

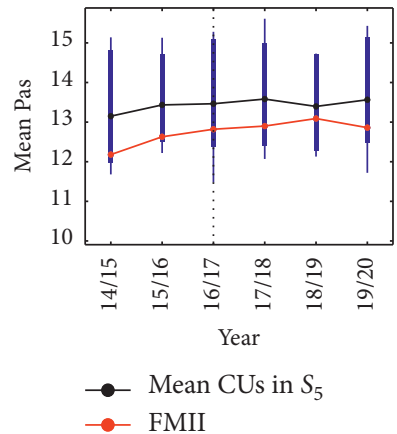

(s)

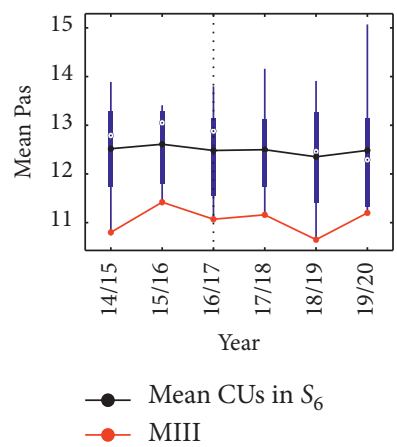

(w)

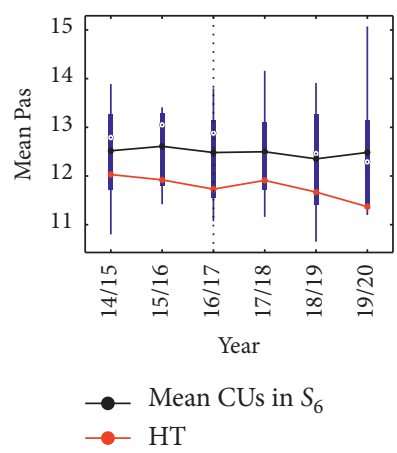

(aa)

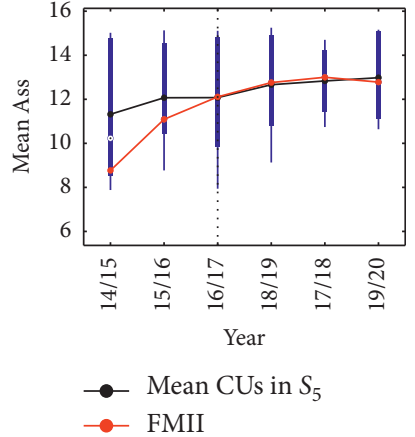

(t)

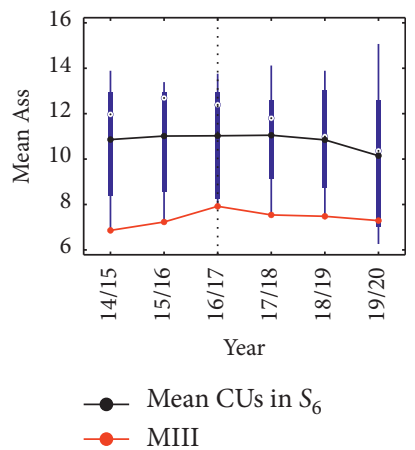

(x)

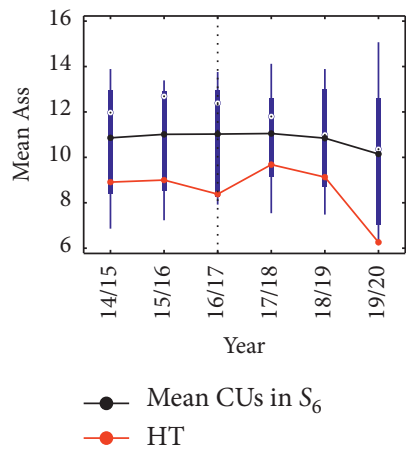

(ab)

FIgURE 1: Performance indices of the CUs versus time. The red lines represent the values of the performance indices of each indicated CU and the black lines represent the arithmetic means of the performance indices of the CUs in the same semester, either $\mathbf{S}_{2}, \ldots, \mathbf{S}_{6}$, respectively: (a) \%Pas/Enr vs. year; (b) \%Pas/Ass vs. year; (c) Mean Pas vs. year; (d) Mean Ass vs. year; (e) \%Pas/Enr vs. year; (f) \%Pas/Ass vs. year; (g) Mean Pas vs. year; (h) Mean Ass vs. year; (i) \%Pas/Enr vs. year; (j) \%Pas/Ass vs. year; (k) Mean Pas vs. year; (l) Mean Ass vs. year; (m) \%Pas/Enr vs. year; (n) \%Pas/Ass vs. year; (o) Mean Pas vs. year; (p) Mean Ass vs. year; (q) \%Pas/Enr vs. year; (r) \%Pas/Ass vs. year; (s) Mean Pas vs. year; (t) Mean Ass vs. year; (u) \%Pas/Enr vs. year; (v) \%Pas/Ass vs. year; (w) Mean Pas vs. year; (x) Mean Ass vs. year; (y) \%Pas/Enr vs. year; (z) \%Pas/Ass vs. year; (aa) Mean Pas vs. year; (ab) Mean Ass vs. year.

matrix $\Delta=\left[\delta_{i j}\right]$ of object-to-object (dis)similarities, calculated based on a distance metric. The MDS assigns coordinates to each object in a $p$-dimensional space, with $p \leq q$, and organizes the set in a symmetric matrix $\boldsymbol{\Phi}=\left[\phi_{i j}\right]$ in order to reproduce $\Delta$ as best as possible. This is accomplished by means of a function optimization procedure that tests several configurations. A common index to assess how well a given configuration in $\boldsymbol{\Phi}$ reproduces the original distances matrix $\Delta$ is the raw stress as follows:

$$
R=\left[\varphi_{i j}-f\left(\delta_{i j}\right)\right]^{2},
$$

where $f\left(\delta_{i j}\right)$ stands for a linear or nonlinear function. The smaller the stress, $R$, the better is the fit between $\Phi$ and $\Delta$. For $p \leq 3$, the objects can be represented in a graph that can be analyzed in the perspective of the objects location, meaning that similar objects appear close to each other, while dissimilar objects are located distant from each other. The orientation of the final MDS chart has no special meaning, and the map is insensitive to translation, rotation, and magnification.

For each year, 2014/2015-2019/2020, the data characterizing the CUs in a semester are organized in a $5 \times 4$ array, 
TABle 2: Pearson correlation between each CUs and the semester averages indices.

\begin{tabular}{lcccccc}
\hline & & Starting & \multicolumn{3}{c}{ Pearson correlation $\rho_{\mathbf{x y}}$} \\
CU & Semester & $\begin{array}{c}\text { year } \\
\text { Pas/Enr }\end{array}$ & Pas/Ass & $\begin{array}{c}\text { Mean } \\
\text { Pas }\end{array}$ & $\begin{array}{c}\text { Mean } \\
\text { Ass }\end{array}$ \\
\hline MI & $\mathbf{S}_{2}$ & $2016-2017$ & 0.81 & 0.74 & 0.79 & 0.71 \\
TII & $\mathbf{S}_{3}$ & $2017-2018$ & 0.89 & 0.88 & 0.90 & 0.65 \\
SM & $\mathbf{S}_{4}$ & $2016-2017$ & 0.31 & 0.28 & 0.18 & 0.67 \\
FMI & $\mathbf{S}_{4}$ & $2017-2018$ & 0.73 & 0.30 & 0.62 & 0.32 \\
FMII & $\mathbf{S}_{5}$ & $2016-2017$ & 0.89 & 0.84 & 0.94 & 0.76 \\
MIII & $\mathbf{S}_{6}$ & $2016-2017$ & 0.62 & 0.75 & 0.27 & 0.79 \\
HT & $\mathbf{S}_{6}$ & $2016-2017$ & 0.89 & 0.92 & 0.91 & 0.44 \\
\hline
\end{tabular}

$\widetilde{W}=\left[\widetilde{w}_{i k}\right]$, where $\widetilde{w}_{i k}, i=1, \ldots, 5, k=1, \ldots, 4$, represents the $i^{\text {th }} \mathrm{CU}$ in a given $\mathbf{S}_{2}, \ldots, \mathbf{S}_{6}$ semester and its $k^{\text {th }}$ characterizing index in the set $\{\mathrm{Pas} / \mathrm{Enr}$, Pas/Ass, Mean Pas, Mean Ass $\}$. The array $\widetilde{W}$ is firstly normalized to prevent numerical saturation. Therefore, the columns of $\widetilde{W}$, to be represented by $\widetilde{u}_{k}$, are expressed as follows:

$$
\mathbf{u}_{k}=\frac{\tilde{u}_{k}-\mu\left(\tilde{u}_{k}\right)}{\sigma\left(\widetilde{u}_{k}\right)} .
$$

This leads to a normalized array $\mathbf{W}$, where $\sigma(\bullet)$ represents the standard deviation of the argument. Then, the rows of $\mathbf{W}$, to be represented by $\mathbf{v}_{i}=\left[v_{i 1}, \ldots, v_{i 4}\right]$, are used to compute the matrix $\Delta=\left[\delta_{i j}\right], i, j=1, \ldots, 5$, where $\delta_{i j}$ denotes the Euclidean distance:

$$
\delta_{i j}=\sqrt{\sum_{h=1}^{4}\left(v_{i h}-v_{j h}\right)^{2}} .
$$

Finally, $\Delta$ is processed through the MDS algorithm to generate the maps of objects that represent the CUs in per semester and per year basis.

Figures 2-6 portrait the MDS 3-dimensional maps of the CUs in the semesters $S_{2}, \ldots, S_{6}$ and, for each, in the 6-year period 2014/2015-2019/2020.

Figure 2 unveils the existence of 2 clusters every year. Clusters are separated by dotted lines. Often, one cluster includes $\{\mathrm{MI}, \mathrm{TI}\}$ and the other comprises $\{\mathrm{CPI}, \mathrm{MAII}, \mathrm{S}\}$. Exceptions occur in years 2016/2017 and 2018/2019, where CPI leaves the cluster $\{\mathrm{CPI}, \mathrm{MAII}, \mathrm{S}\}$ and steps into $\{\mathrm{MI}, \mathrm{TI}\}$. Therefore, MI is similar to TI, and the two CUs are quite different from the pair AMII and S. Often, CPI is close to MAII and to $S$ but reveals some variability. In conclusion, over the period 2014/2015-2019/2020, MI did not change its relative positioning with respect to the other CUs in the semester $\mathbf{S}_{2}$, meaning that no effect of the laboratory activities on MI can be noted.

Figure 3 depicts the dynamics of the CUs in semester $\mathbf{S}_{3}$ over the 6-year period 2014/2015-2019/2020. In most years, 3 clusters emerge, namely, $\{$ MAIII, NA, TII $\},\{$ E $\}$, and $\{\mathrm{MII}\}$. $\mathrm{E}$ and MII are CUs that have better and worst results than the others, respectively. In years 2016/2017 and 2019/2020, the CU E enters the cluster \{MAIII, NA, TII\}. Focusing on TII, it can be seen that the CU follows the mainstream. Therefore, TII keeps its relative positioning with respect to the other CUs in the semester $\mathbf{S}_{3}$, meaning, as before, that no effect of the laboratory activities on the CU can be noted.

Figure 4 portraits the MDS maps of the CUs in semester $\mathbf{S}_{4}$. In this case, the behavior of the CUs is almost random, which inhibits any attempt to interpret their dynamics in the period 2014/2015-2019/2020.

In Figure 5, we verify the existence of 2 clusters every year. One includes $\{\mathrm{CADM}, \mathrm{NMM}\}$, and the other comprises $\{$ FMII, FPS, SMI . These clusters correspond to CUs with the best and the worst results in the semester $S_{5}$. The patterns formed by the CUs are stable over the period 2014/ 2015-2019/2020. Thus, regarding FMII, it means that the effect of the laboratory activities cannot be distinguished.

Figure 6 depicts the dynamics of the CUs in semester $\mathbf{S}_{6}$ over the 6-year period 2014/2015-2019/2020. In most years, 2 clusters emerge, namely, $\{\mathrm{HT}, \mathrm{MIII}\}$ and $\{\mathrm{IAS}, \mathrm{IS}, \mathrm{MPI}\}$. The exception is years 2017/2018 and 2018/2019, where IAS and IS change a bit. The CUs HT and MIII are always close to each other, but they change their loci in year 2019/2020, meaning that MIII seems to move towards the mainstream, which translates into improving its results.

In synthesis, the multivariate analysis performed unveils some evidences about the behavior of the CUs, but in most cases the results are still somewhat inconclusive.

\subsection{MDS with Procrustes Data Clustering and Visualization.} To improve the visualization and ease the detection of possible relationships between the data, each set of maps depicted in Figures 2-6 can be replaced by one chart that collapses all information embedded in the set. Hereafter, this is accomplished by means of Procrustes analysis. Procrustes is a numerical technique that takes a collection of shapes and transforms them for maximum superposition, using the linear transformations $\mathbf{O}=\{$ translation, reflection, orthogonal rotation, magnification/reduction of size $][29,32-34]$.

For the CUs in each semester $\mathbf{S}_{2}, \ldots, \mathbf{S}_{6}$, Procrustes involves four iterative steps: (i) the user selects a reference MDS map (in the present case, the one corresponding to the year 2014/2015 is adopted but any available instance can be used); (ii) the algorithm superimposes all remaining maps into the current reference using the transformations in $\mathbf{O}$; (iii) it computes the 'mean form' of the current set of superimposed maps; and (iv) it calculates the distance between the "mean form" and the reference instances and compares such distance with a given threshold value. If the distance is greater than the threshold, then the algorithm sets the reference to the "mean form" and steps to (ii). Otherwise, it keeps the reference and steps to (ii). At the end, a global representation of all maps that best conforms them is obtained. 


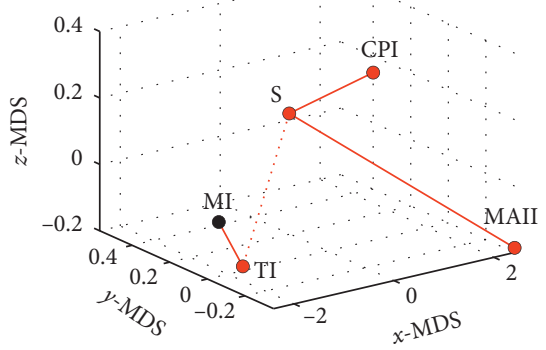

(a)

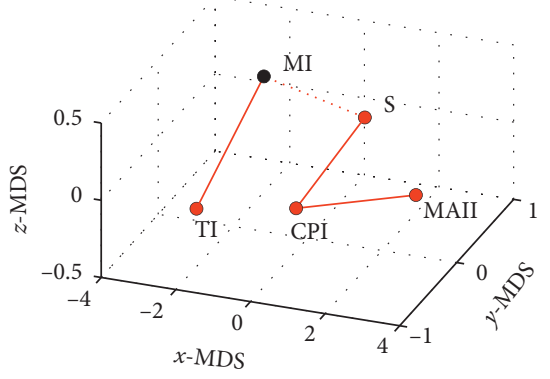

(d)

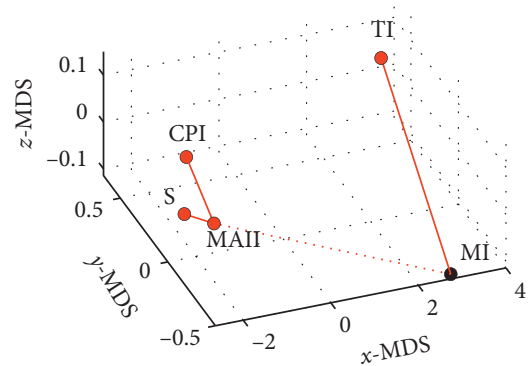

(b)

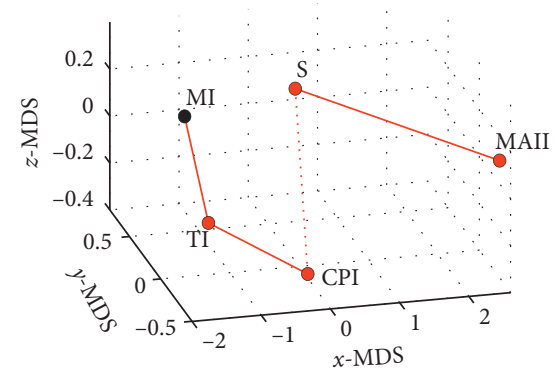

(e)

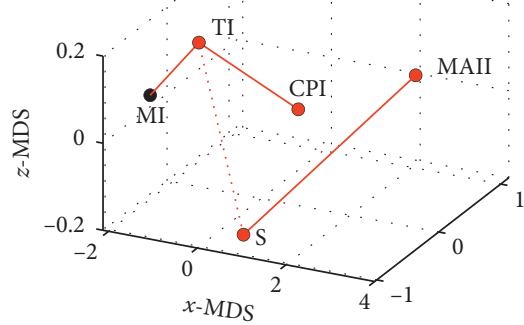

(c)

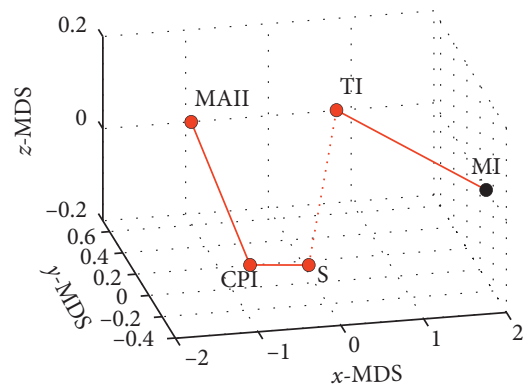

(f)

Figure 2: The MDS 3D maps of the CUs of the semester $\boldsymbol{S}_{2}$ in the 6-year period 2014/2015-2019/2020.

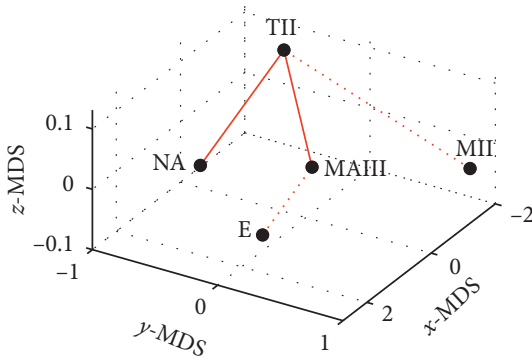

(a)

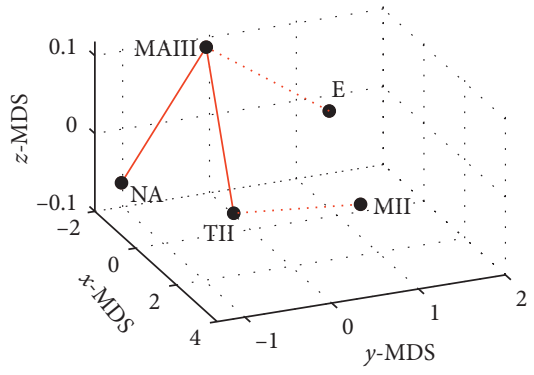

(d)

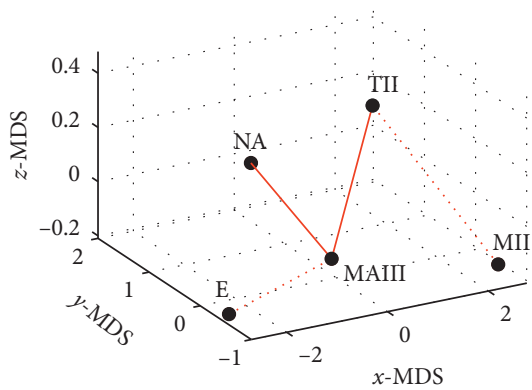

(b)

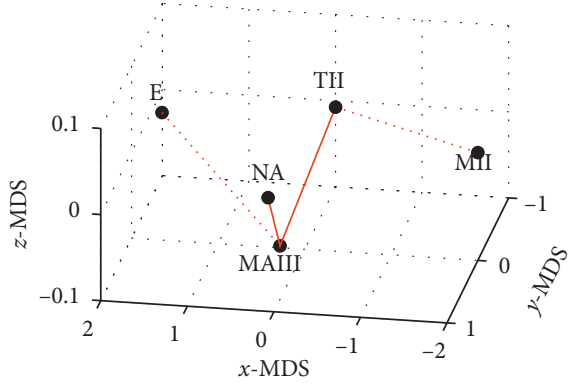

(e)

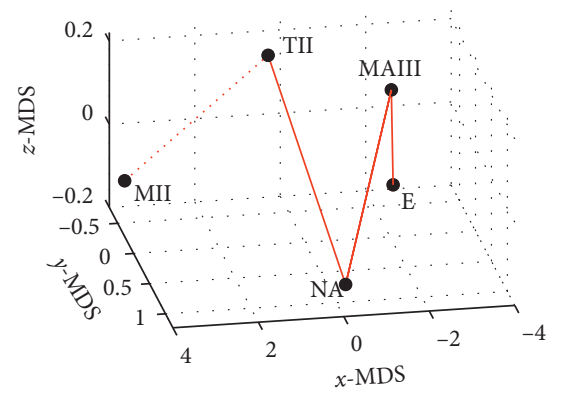

(c)

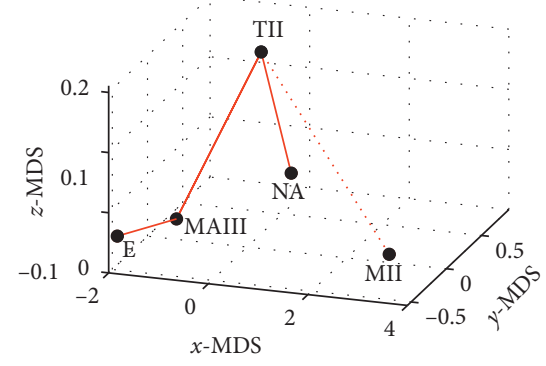

(f)

FIgURE 3: The MDS 3D maps of the CUs of the semester $\boldsymbol{S}_{3}$ in the 6-year period 2014/2015-19/2020.

Figures 7-11 depict the MDS 3D maps of the CUs in each semester $\mathbf{S}_{2}, \ldots, \mathbf{S}_{6}$ obtained by Procrustes. For good readability, circles denote CUs in a given year of the period of time 2014/2015-2019/2020. Square and star marks indicate the first and last year of the interval. The arrows represent the flow of time.
The results obtained are consistent with those in Subsection 4.1, but further conclusions can be drawn, with the advantage of having just one MDS map to interpret:

(i) Figure 7 depicts the map of the CUs in semester $\mathbf{S}_{2}$. The right- and left-hand sides of the chart are 


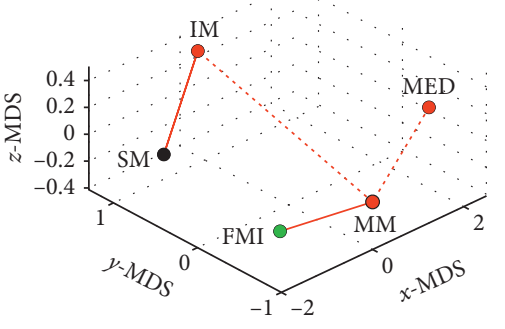

(a)

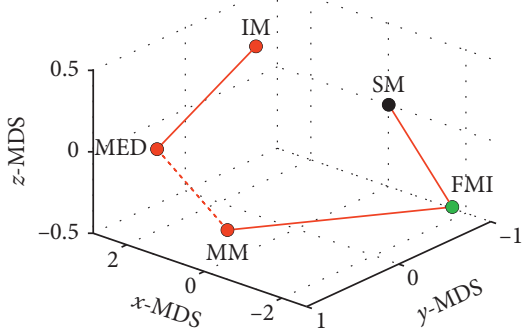

(d)

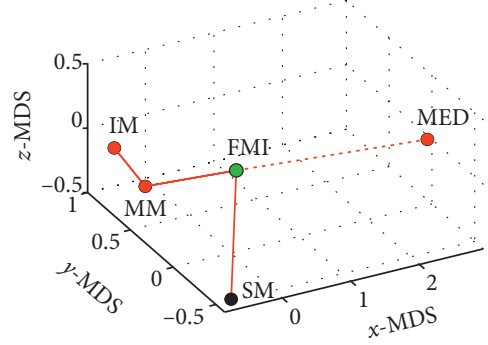

(b)

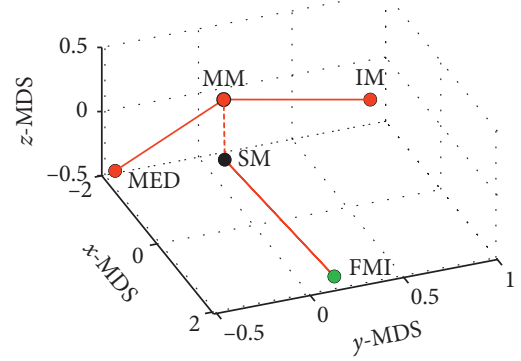

(e)

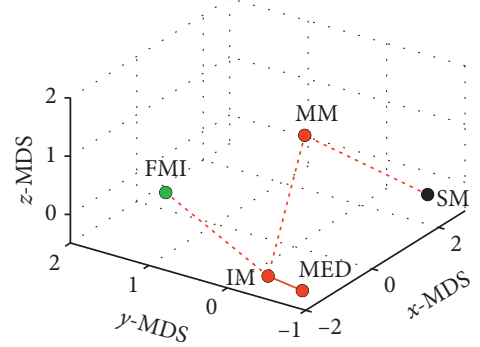

(c)

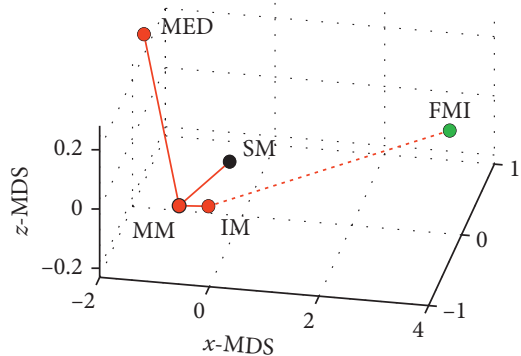

(f)

Figure 4: The MDS 3D maps of the CUs of the semester $\mathbf{S}_{4}$ in the 6-year period 2014/2015-2019/2020.

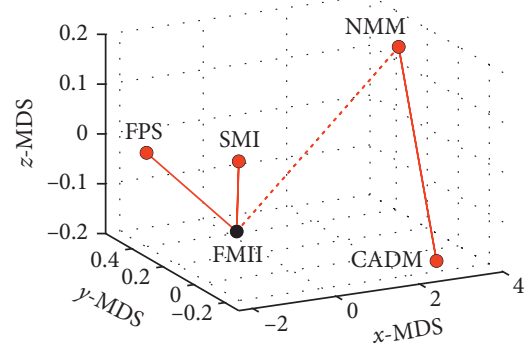

(a)

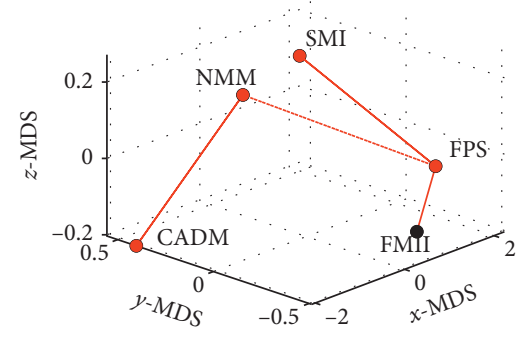

(d)

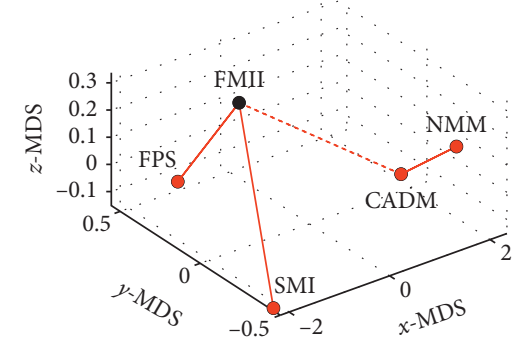

(b)

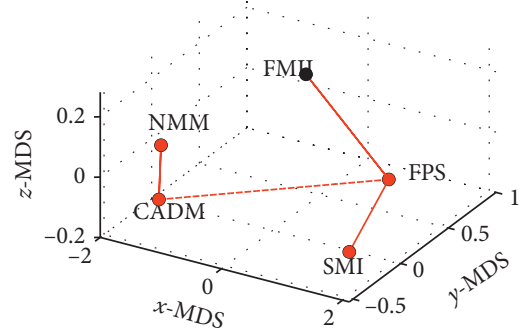

(e)

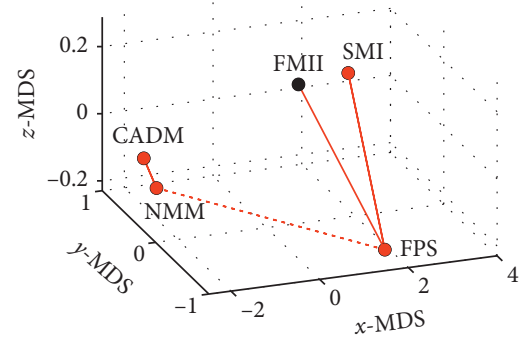

(c)

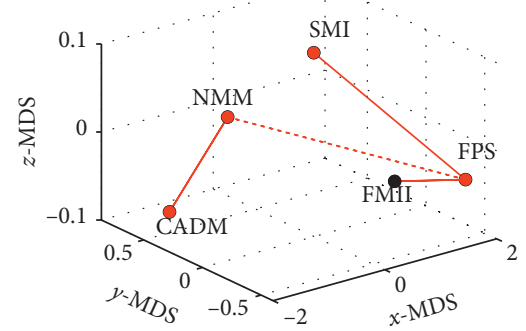

(f)

Figure 5: The MDS 3D maps of the CUs of the semester $\mathbf{S}_{5}$ in the 6-year period 2014/2015-2019/2020.

populated with the CUs with best $\{$ MAII, S $\}$ and worst $\{\mathrm{MI}, \mathrm{TI}\}$ results, respectively. In the middle, PCI emerges as a CU with intermediate results. It can be seen that, in some years, PCI approaches the left- or the right-hand sides of the map. The CU MI evolves in time, describing a path in the MDS, but it does not move toward the right-hand side, meaning that the results in the UC are not improving; (ii) The CUs in $\mathbf{S}_{3}$ are portrait in Figure 8. In the rightand left-hand sides of the chart are the CUs with best $\{E\}$ and worst $\{\mathrm{MII}\}$ results, respectively. In the middle of the chart are the CUs with intermediate results $\{\mathrm{MAIII}, \mathrm{NA}, \mathrm{TII}\}$. It can be seen that TII describes a path with a certain amplitude but remains in the central region of the MDS, meaning that no significant changes can be noticed on the results over the period 2014/2015-2019/2020. The 


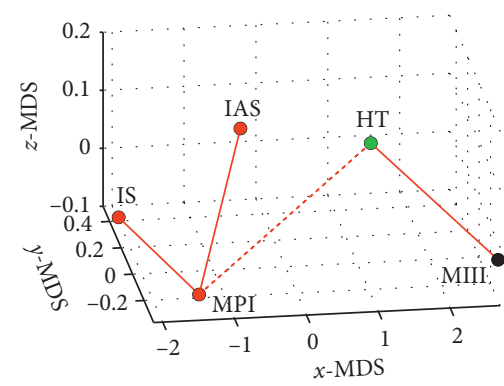

(a)

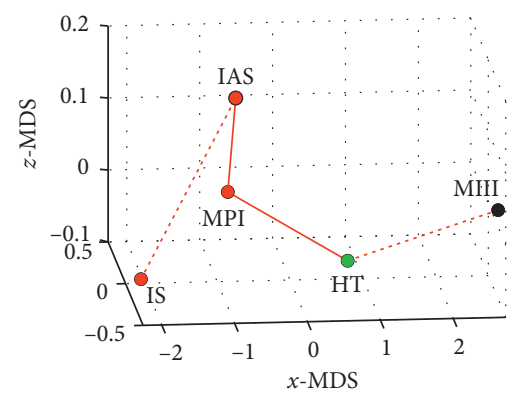

(d)

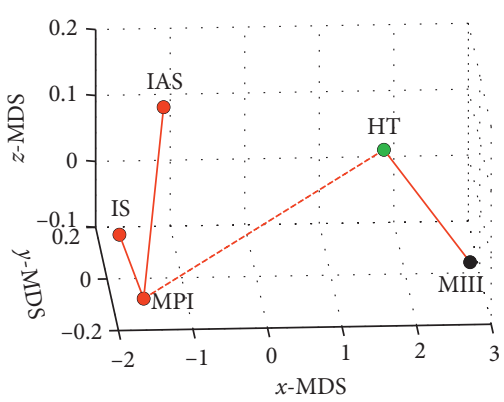

(b)

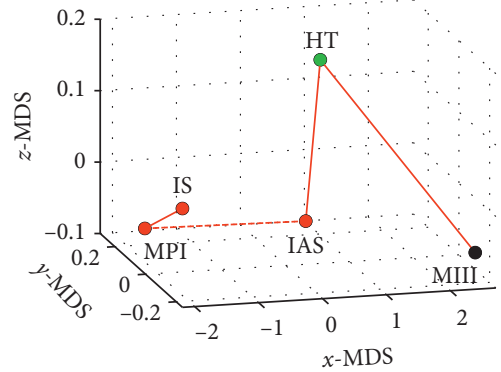

(e)

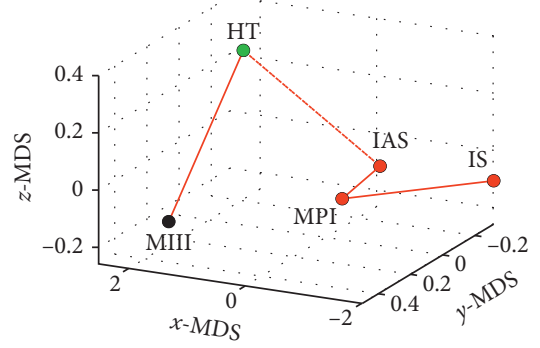

(c)

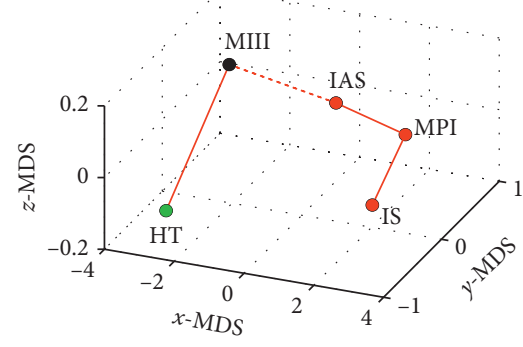

(f)

Figure 6: The MDS 3D maps of the CUs of the semester $\mathbf{S}_{6}$ in the 6-year period 2014/2015-2019/2020.

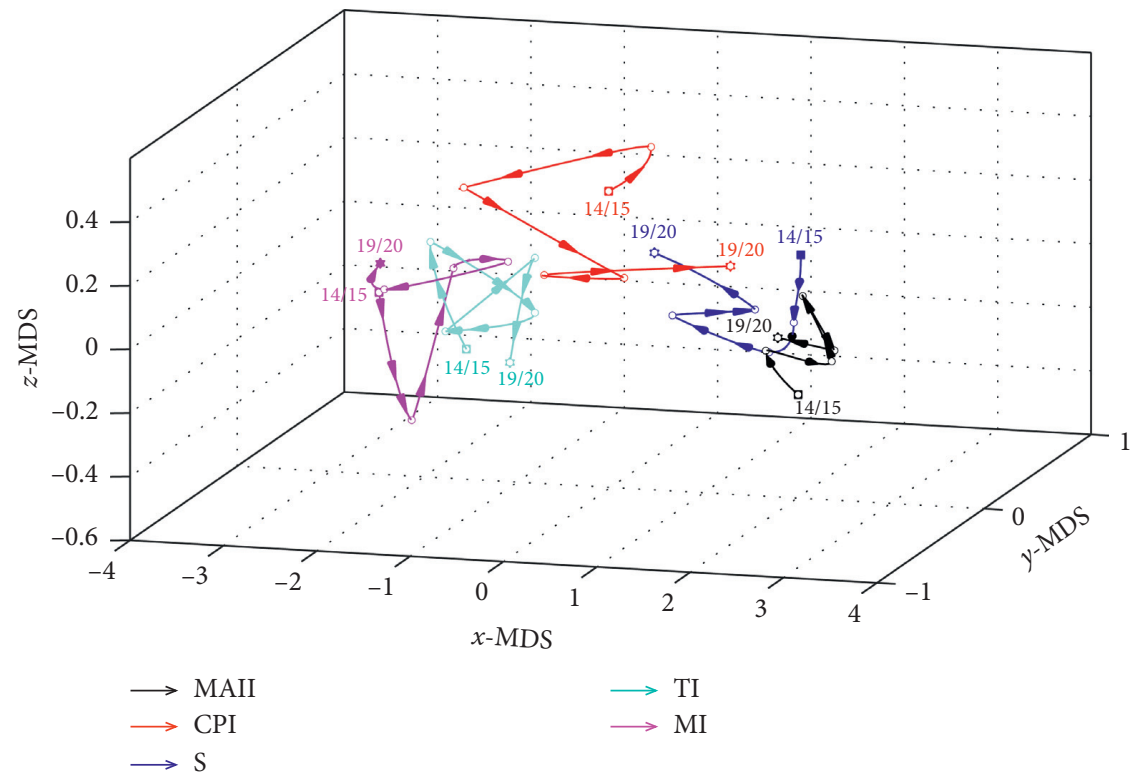

Figure 7: The MDS 3D maps of the CUs in semester $\mathbf{S}_{2}$, superimposed by means of Procrustes for the 6-year period 2014/2015-2019/2020. Each circle denotes a CU in a given year. Square and star marks indicate the first and last year of the interval. The arrows represent the flow of time.

CU MII evolves within a small volume on the lefthand side, away from the CUs with intermediate and good results. This means that this UC has results clearly worse than the others and should be the focus of special attention;

(iii) Figure 9 depicts the map of the CUs in semester $\mathbf{S}_{4}$. The CUs with best results $\{\mathrm{IM}, \mathrm{MED}, \mathrm{MM}\}$ are placed at the top-left region of the MDS. The CU
FMI evolves on the right-hand side, approaching $\{I M, M E D, M M\}$ in the first years and, then, moving away in the most recent years of the period 2014/ 2015-2019/2020. The CU SM starts on the bottom of the map and approaches the set $\{\mathrm{IM}, \mathrm{MED}, \mathrm{MM}\}$, especially from the year 2017/2018 onwards. Therefore, it can be concluded that MFI is not improving, but SM is getting better results; 


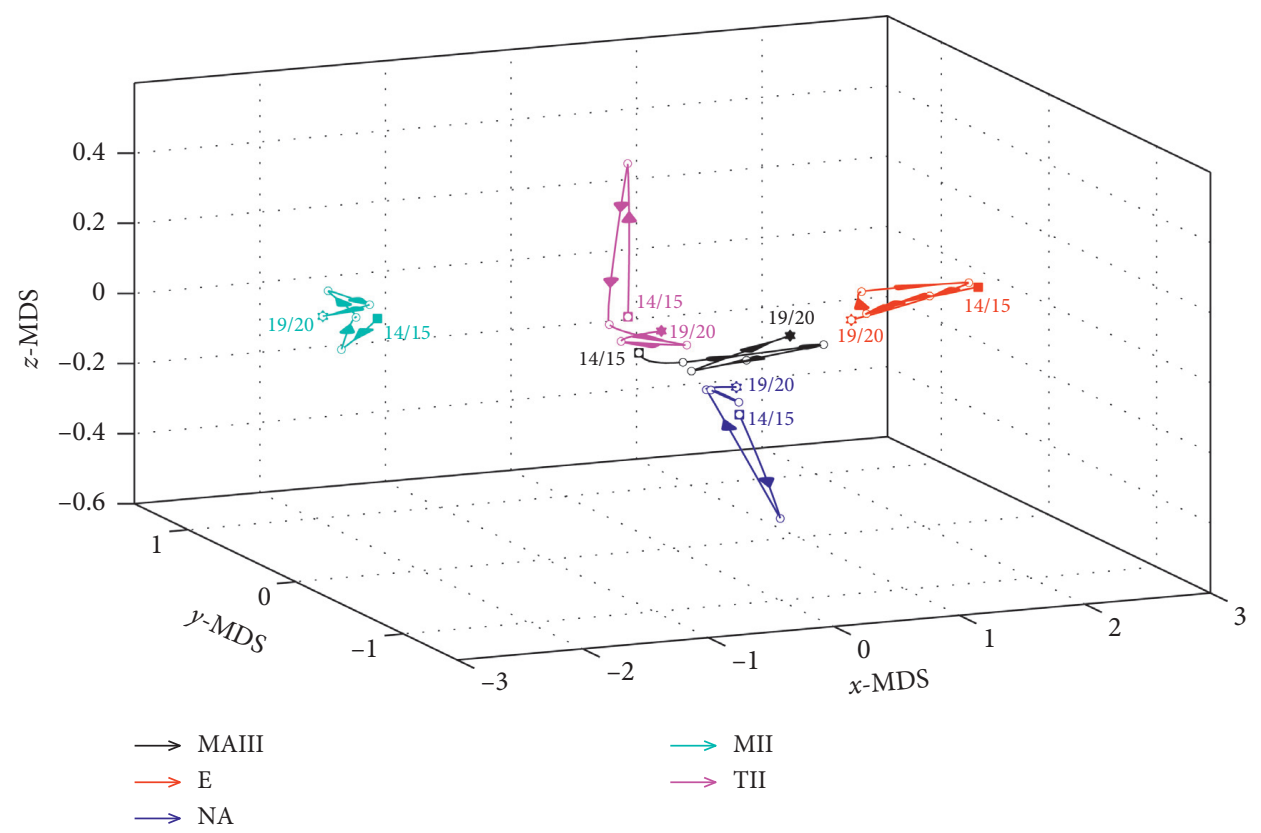

Figure 8: The MDS 3D maps of the CUs in semester $\mathbf{S}_{3}$, superimposed by means of Procrustes for the 6-year period 2014/2015-2019/2020. Each circle denotes a CU in a given year. Square and star marks indicate the first and last year of the interval. The arrows represent the flow of time.

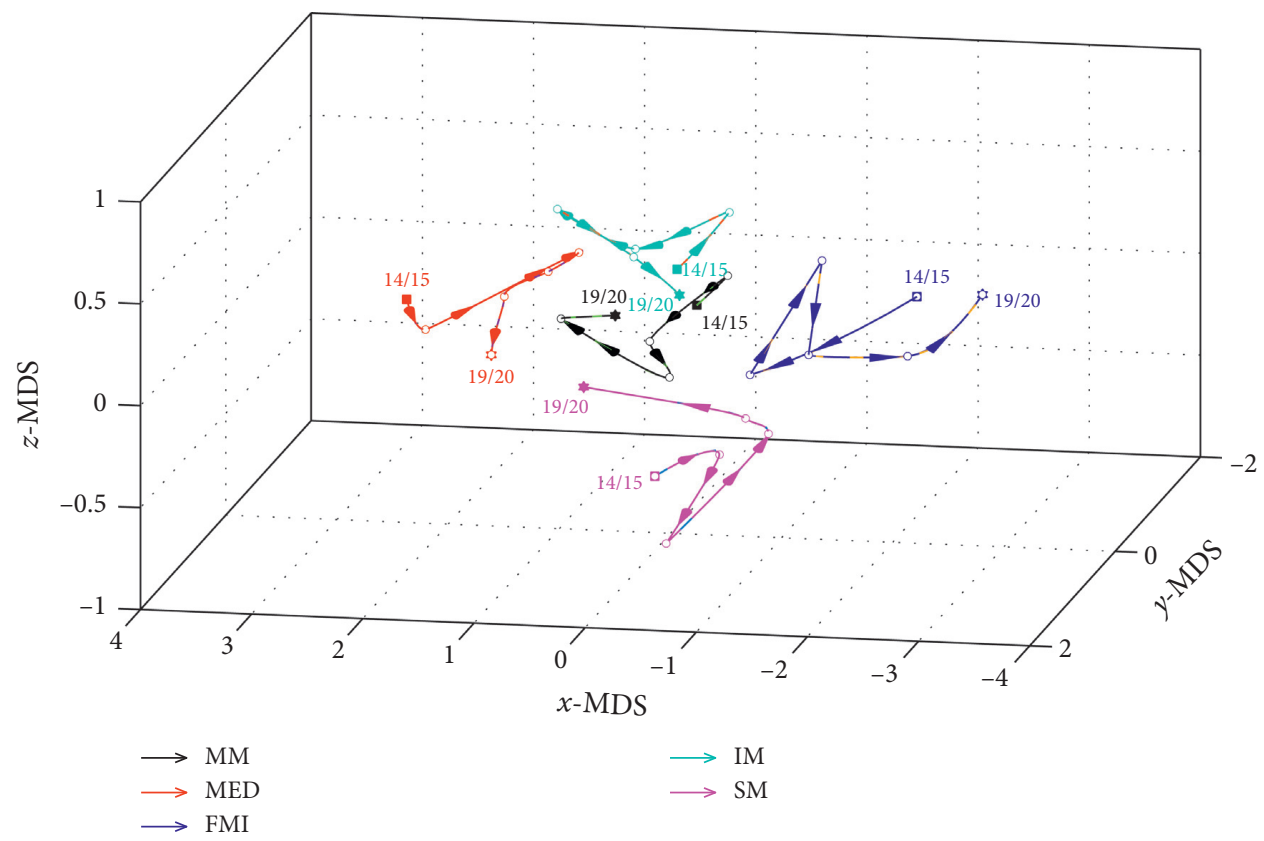

Figure 9: The MDS 3D maps of the CUs in semester $\mathbf{S}_{4}$, superimposed by means of Procrustes for the 6-year period 2014/2015-2019/2020. Each circle denotes a CU in a given year. Square and star marks indicate the first and last year of the interval. The arrows represent the flow of time.

(iv) In Figure 10, which portraits the dynamics of the CUs in semester $S_{5}$, it can be seen that there are 2 CUs with much better performance than the others. The best $\{\mathrm{CADM}, \mathrm{NMM}\}$ are on the right-hand side, while the worst $\{$ FMII, FPS, SMI\} evolve on the lefthand side of the MDS map. Regarding FMII, it seems to exhibit a timid movement toward the set
\{CADM, NMM , which translates into a slight improvement on the results in the UC;

(v) Figure 11 shows the MDS of the CUs in semester $\mathbf{S}_{6}$. The right- and left-hand sides of the chart are populated with the CUs with worst $\{\mathrm{HT}, \mathrm{MIII}\}$ and best $\{\mathrm{IS}\}$ results, respectively. The middle region is occupied by the CUs that have intermediate results 


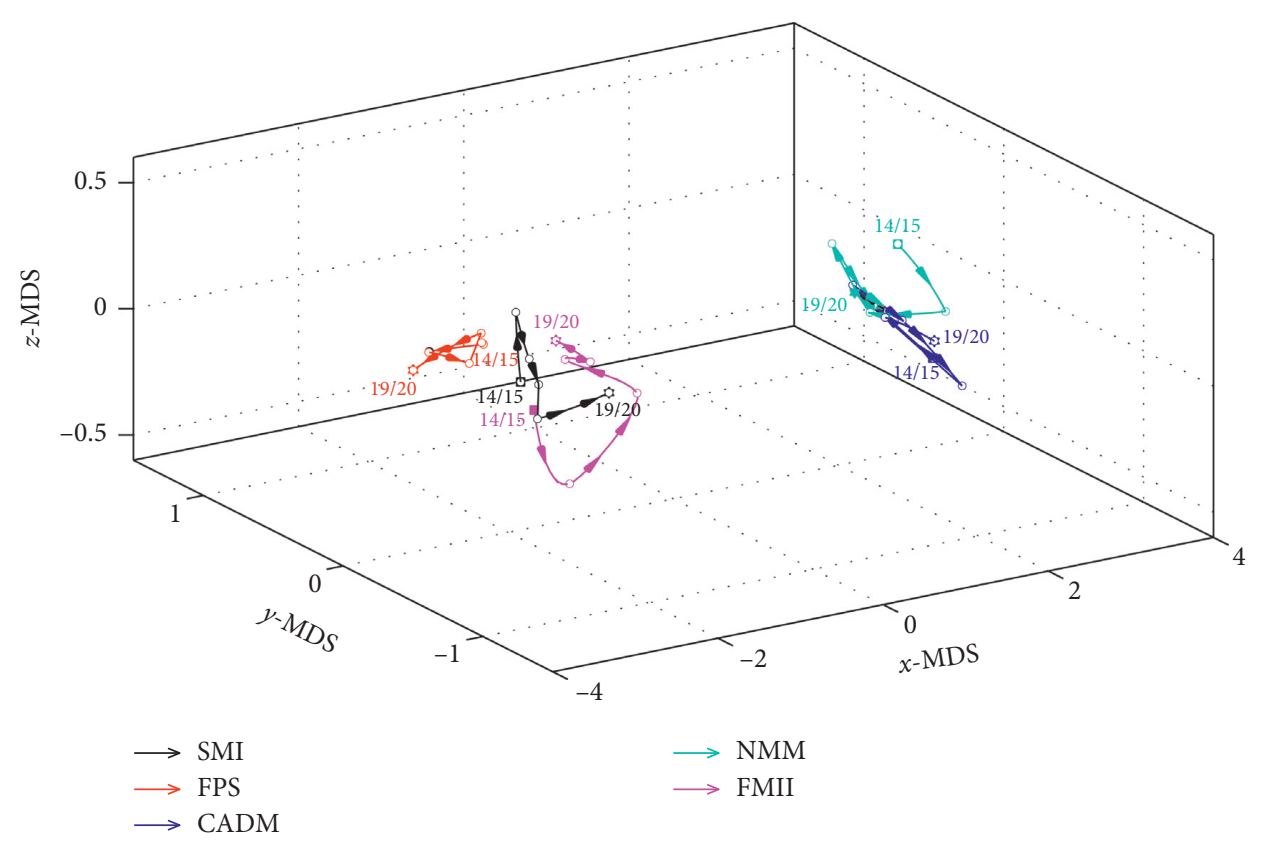

Figure 10: The MDS 3D maps of the CUs in semester $\mathbf{S}_{5}$, superimposed by means of Procrustes for the 6-year period 2014/2015-2019/2020. Each circle denotes a CU in a given year. Square and star marks indicate the first and last year of the interval. The arrows represent the flow of time.

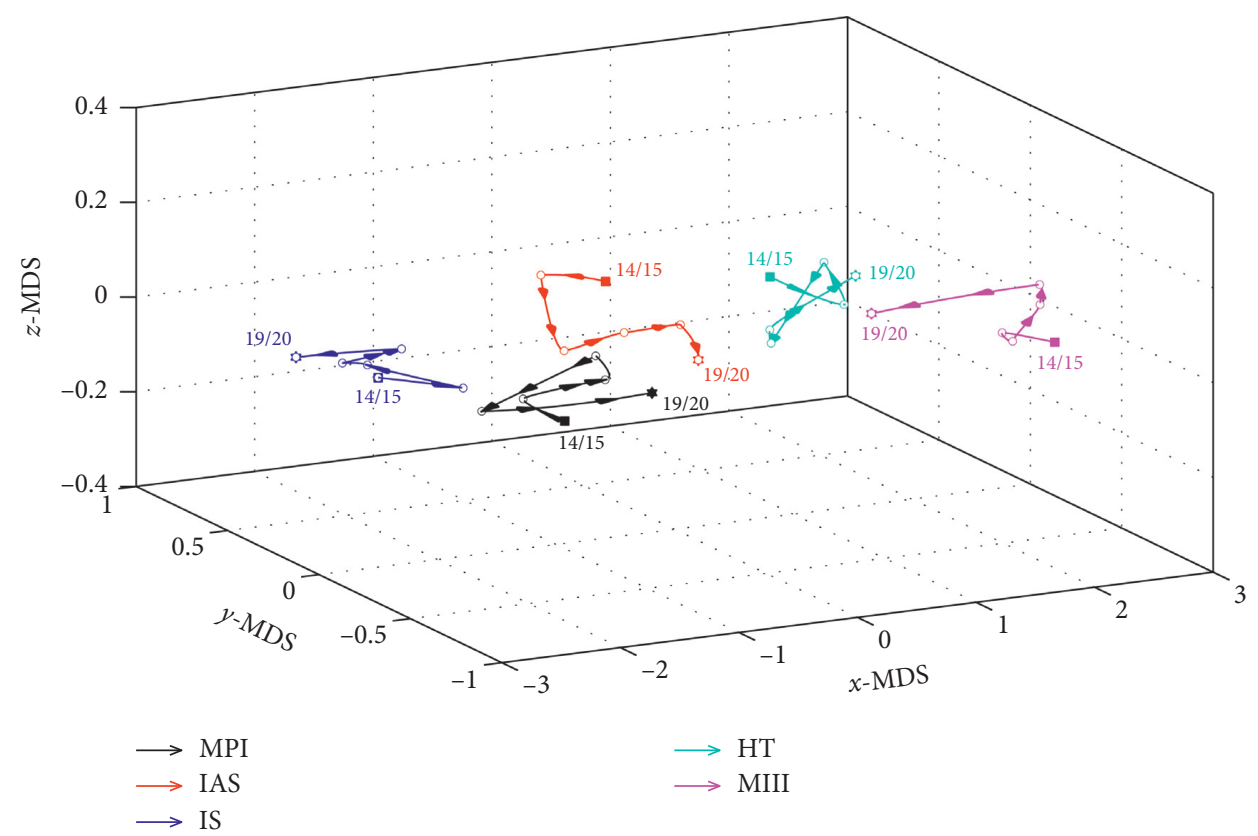

FIGURe 11: The MDS 3D maps of the CUs in semester $\mathbf{S}_{6}$, superimposed by means of Procrustes for the 6-year period 2014/2015-2019/2020. Each circle denotes a CU in a given year. Square and star marks indicate the first and last year of the interval. The arrows represent the flow of time.

\{IAS, MPI\}. It can be seen that HT evolves within a relative small volume, with no significant variation on the results obtained over the period 2014/ 2015-2019/2020. The CU MIII evolves approaching the middle of the map, meaning that the results are getting better.

\section{Conclusions}

The effect of laboratory activities implemented in 7 core CUs of the MIEM degree at FEUP was investigated in the perspective of the results achieved by the students. The CUs were characterized by 4 performance indices in the 6-year 
time horizon 2014/2015-2019/2020. In a first phase, the analysis of single indices revealed inconclusive and pointed towards the need of using multivariate analytical models to compare and visualize the data. In a second phase, the CUs were considered as objects defined in the 4-dimensional space of features, and the MDS was adopted for dimensionality reduction, clustering, and data visualization. Finally, Procrustes processing was used to achieve meaningful maps of objects which are able to unveil all hidden relationships between the data. The MDS and Procrustes showed that, for now, the impact of the laboratory activities in the results of most CUs is insipient. However, for SM, FMII, and MIII, the effect seems to be positive since the results in these CUs have been improving in the last few years. Nevertheless, a longer period of analysis is needed to reach more solid conclusions.

\section{Data Availability}

The data used to support the findings of this study are available from the author upon request.

\section{Conflicts of Interest}

The authors declare that they have no conflicts of interest.

\section{References}

[1] R. V. Krivickas and J. Krivickas, "Laboratory instruction in engineering education," Global Journal of Engineering Education, vol. 11, pp. 191-196, 2007.

[2] L. D. Feisel and A. J. Rosa, "The role of the laboratory in undergraduate engineering education," Journal of Engineering Education, vol. 94, no. 1, pp. 121-130, 2005.

[3] D. A. Kolb, Experiential Learning: Experience as the Source of Learning and Development, Prentice-Hall, Hoboken, NJ, USA, 2014.

[4] D. S. Domin, "A review of laboratory instruction styles," Journal of Chemical Education, vol. 76, no. 4, p. 543, 1999.

[5] S. Lal, A. D. Lucey, E. D. Lindsay et al., "Student perceptions of instruction sheets in face-to-face and remotely-operated engineering laboratory learning," European Journal of Engineering Education, vol. 45, no. 4, pp. 491-515, 2020.

[6] R. T. Bye and O. L. Osen, "On the development of laboratory projects in modern engineering education," in Proceedings of the 2019 IEEE Global Engineering Education Conference (EDUCON), pp. 1300-1307, Dubai, UAE, April 2019.

[7] J. Li, S.-h. Han, and S. Fu, "Exploring the relationship between students' learning styles and learning outcome in engineering laboratory education," Journal of Further and Higher Education, vol. 43, no. 8, pp. 1064-1078, 2019.

[8] M. C. Reese, "Comparison of student achievement among two science laboratory types: traditional and virtual," Doctoral dissertation, Mississippi State University, Mississippi, MS, USA, 2020.

[9] A. Van den Beemt, M. MacLeod, J. Van der Veen et al., "Interdisciplinary engineering education: a review of vision, teaching, and support," Journal of Engineering Education, vol. 109, no. 3, pp. 508-555, 2020.

[10] K. Wright, P. E. Slaboch, and R. Jamshidi, "Technical writing improvements through engineering lab courses," International Journal of Mechanical Engineering Education, 2020.
[11] A. Hofstein and V. N. Lunetta, "The role of the laboratory in science teaching: neglected aspects of research," Review of Educational Research, vol. 52, no. 2, pp. 201-217, 1982.

[12] W. J. Leonard, R. J. Dufresne, and J. P. Mestre, "Using qualitative problem-solving strategies to highlight the role of conceptual knowledge in solving problems," American Journal of Physics, vol. 64, no. 12, pp. 1495-1503, 1996.

[13] D. Urbano, "Effectiveness of traditional laboratory classes to learn basic concepts of electric circuits: a case study," in Proceedings of the International Conference on Interactive Collaborative Learning, pp. 693-701, Springer, Budapest, Hungary, September 2017.

[14] C. Wieman and N. G. Holmes, "Measuring the impact of an instructional laboratory on the learning of introductory physics," American Journal of Physics, vol. 83, no. 11, pp. 972-978, 2015.

[15] P. Xia, A. M. Lopes, and M. T. Restivo, "A review of virtual reality and haptics for product assembly (part 1): rigid parts," Assembly Automation, vol. 33, no. 1, pp. 68-77, 2013.

[16] P. Xia, A. Mendes Lopes, and M. T. Restivo, "A review of virtual reality and haptics for product assembly: from rigid parts to soft cables," Assembly Automation, vol. 33, no. 2, pp. 157-164, 2013.

[17] M. T. Restivo, A. M. Lopes, and P. J. Xia, “"Feeling” Young modulus of materials," in Proceedings of the 2012 9th International Conference on Remote Engineering and Virtual Instrumentation (REV), pp. 1-4, Bilbao, Spain, July 2012.

[18] M. T. Restivo, J. Mendes, A. M. Lopes, C. M. Silva, and F. Chouzal, "A remote laboratory in engineering measurement," IEEE Transactions on Industrial Electronics, vol. 56, no. 12, pp. 4836-4843, 2009.

[19] M. T. Restivo, A. M. Lopes, L. dos Santos Machado, and R. M. De Moraes, "Adding tactile information to remote virtual labs," in Proceedings of the 2011 IEEE Global Engineering Education Conference (EDUCON), pp. 1120-1124, Amman, Jordan, April 2011.

[20] K. Thorne, Blended Learning: How to Integrate Online Traditional Learning, Kogan Page Publishers, London, UK, 2003.

[21] C. J. Bonk and C. R. Graham, The Handbook of Blended Learning: Global Perspectives, Local Designs, John Wiley \& Sons, San Francisco, CA, USA, 2012.

[22] H. Abdi and L. J. Williams, "Principal component analysis," Wiley Interdisciplinary Reviews: Computational Statistics, vol. 2, no. 4, pp. 433-459, 2010.

[23] Y. V. Tan and J. Roy, "Bayesian additive regression trees and the General BART model," Statistics in Medicine, vol. 38, no. 25, pp. 5048-5069, 2019.

[24] J. T. Machado and A. M. Lopes, “Artistic painting: a fractional calculus perspective," Applied Mathematical Modelling, vol. 65, pp. 614-626, 2019.

[25] M. Sellbom and A. Tellegen, "Factor analysis in psychological assessment research: common pitfalls and recommendations," Psychological Assessment, vol. 31, no. 12, pp. 1428-1441, 2019.

[26] P. Mistry, G. C. Bora, and G. Bora, "Development of yield forecast model using multiple regression analysis and impact of climatic parameters on spring wheat," International Journal of Agricultural and Biological Engineering, vol. 12, no. 4, pp. 110-115, 2019.

[27] A. Afifi, S. May, R. Donatello, and V. A. Clark, Practical Multivariate Analysis, CRC Press, Boca Raton, FL, USA, 2019.

[28] A. M. Lopes and J. A. Tenreiro Machado, "Analysis of temperature time-series: embedding dynamics into the MDS 
method," Communications in Nonlinear Science and $\mathrm{Nu}$ merical Simulation, vol. 19, no. 4, pp. 851-871, 2014.

[29] J. Tenreiro Machado, A. Lopes, and A. Galhano, "Multidimensional scaling visualization using parametric similarity indices," Entropy, vol. 17, no. 4, pp. 1775-1794, 2015.

[30] N. Saeed, H. Nam, M. I. U. Haq, and D. B. Muhammad Saqib, "A survey on multidimensional scaling," ACM Computing Surveys, vol. 51, no. 3, pp. 1-25, 2018.

[31] A. M. Lopes and J. A. Tenreiro Machado, "Integer and fractional-order entropy analysis of earthquake data series," Nonlinear Dynamics, vol. 84, no. 1, pp. 79-90, 2016.

[32] J. C. Gower and G. B. Dijksterhuis, Procrustes Problems, Oxford University Press, Oxford, UK, 2004.

[33] A. M. Lopes, J. A. Tenreiro Machado, and A. M. Galhano, "Multidimensional scaling visualization using parametric entropy," International Journal of Bifurcation and Chaos, vol. 25, no. 14, p. 1540017, 2015.

[34] J. T. Machado and A. M. Lopes, "Multidimensional scaling locus of memristor and fractional order elements," Journal of Advanced Research, vol. 25, pp. 147-157, 2020. 